\section{Vielversprechende Landkarte}

\author{
Seit Anfang der 90er-Jahre hat das Konzept des Stoffstrommanagements Ein- \\ zug in die deutsche Umweltdebatte gehalten. Stark inspiriert durch die Diskus- \\ sion in den Niederlanden, hat darauf aufbauend insbesondere die Enquete- \\ Kommission "Schutz des Menschen und der Umwelt" des Deutschen \\ Bundestages den Ansatz vorangetrieben. Das Konzept ist ein Versuch zur Ope- \\ rationalisierung des Leitbildes einer nachhaltigen Entwicklung, der in einem \\ engen Zusammenhang mit den Strategien einer integrierten Produktpolitik \\ steht. Wo steht diese Diskussion heute und welche Konsequenzen für die Wei- \\ terentwicklung eröffnen sich daraus?
}

$\mathrm{D}$ Von Karl-Otto Henseling, atz des Stoffstrommanagements erhebt den Anspruch, eine neue, integrierte Perspektive in die bisher um die Regulierung von Stoffen, die Gestaltung von Produkten, aber auch die Ökologisierung von Anwendungsverhalten geführte Debatte zu bringen. Im Vordergrund steht dabei ein kooperativer Politikstil, der auf eine stärkere Aufgabenteilung und Selbstorganisation der Akteure im Sinne proaktiver und präventiver Maßnahmen setzt.

Um den Stand eines solchen ehrgeizigen Projektes zu beurteilen, ist die Betrachtung unterschiedlicher Ebenen notwendig. Es gilt vor allem folgende Fragen zu klären:

- Gibt es eine gemeinsame Vorstellung über das mit dem Ansatz des Stoffstrommanagements verbundene Ziel einer nachhaltigen Gestaltung von Stoffströmen?

- Wie ist der Stand der Methoden und Instrumente, die insbesondere auch in der betrieblichen Praxis und in konkreten Projekten eingesetzt werden und wie wird dies gefördert?

Auf diese Fragen wird der Beitrag in komprimierter Form eingehen und damit versuchen, sowohl eine aktuelle Landkarte des Stoffstrommanagements zu skizzieren als auch Folgerungen für das weitere Handeln abzuleiten.

\section{- Normative Grundlagen}

Die Ziele und normativen Grundlagen des Stoffstrommanagements sind zwei recht unterschiedlichen Bereichen zuzuordnen. Einerseits besteht eine enge Verbindung zur Nachhaltigkeitsdiskussion und den von der Enquete-Kommission „Schutz des Menschen und der Umwelt“ 1994 formulierten ökologischen Grundregeln einer nachhaltigen Entwicklung (1). Andererseits werden in konkreten Projekten sehr pragmatische Ziele verfolgt, die ihren Ursprung in abfallwirtschaftlichen Fragen, bei betrieblichen Umweltzielen oder umweltbezogenen Produktanforderungen haben. Bisher sind diese beiden Zielebenen noch wenig vermittelt. Erste Arbeiten zur methodischen Verknüpfung werden im Rahmen des Bedürfnisfeldansatzes geleistet (2).

Die Vorstellungen über eine nachhaltige, das heißt dauerhaft umweltgerechte Gestaltung von Stoffströmen sind im Heft 62 der Zeitschrift Politische Ökologie (September 1999) breit diskutiert worden. Rainer Grießhammer beschreibt darin die Entwicklung der politischen Diskussion von der am „Schadstoff des Monats“ orientierten Chemiepolitik der 70er und 80er Jahre zu einer auf die nachhaltige Gestaltung von Stoffströmen durch Innovationen zielenden Umweltpolitik (3). enz, Suffizienz und Konsistenz. Unter Konsistenz wird dabei die Verträglichkeit von natürlichen und industriellen Stoffströmen verstanden. Sie wird dadurch erreicht, dass industrielle Stoffströme entweder störsicher im abgeschlossenen Eigenkreislauf geführt werden oder sich in die Stoffwechselprozesse der umgebenden Natur problemlos einfiugen (4). Nachhaltiges Wirtschaften bedeutet vor allem den Erhalt der Produktivität und der Reproduktionsfähigkeit der Natur (5). Es besteht zunehmend Einigkeit, dass es keine eindimensionale Lösung gibt: Der Weg zu nachhaltigen Stoffströmen wird eine Mischung aus effizienter und/oder konsistenter Stoffstromgestaltung sowie suffizienten Pro-
Gängige Stichworte dieser Diskussion sind Effizi- duktnutzungsmustern sein. Die Bedürfnisfelddiskussion ist eine konsequente Antwort auf diese Situation. Sie ermöglicht es, bedürfnisfeldspezifische Vorstellungen über nachhaltige Stoffströme zu entwickeln, die konkrete Handlungsperspektiven eröffnen.

Bei der Debatte um die Umweltqualitäts- und -handlungsziele im Stoffstrommanagement fehlen noch weitgehend Ansätze zur Verknüpfung nationaler oder internationaler Zielvorgaben mit der einzelwirtschaftlichen Ebene. Wie kann sichergestellt werden, dass das Erreichen von stoffstromorientierten Unternehmenszielen auch ökologische Effektivität bzw. Richtungssicherheit des Gesamtsystems gewährleistet?

\section{Methoden und Instrumente}

Auf der Ebene des innerbetrieblichen Stoffstrommanagements, die von der eher weiten Definition von Stoffstrommanagement durch die Enquete-Kommission ebenfalls abgedeckt wird, gibt es einen sehr ausdifferenzierten Methodenkasten, der als Weiterentwicklung des betrieblichen Umweltmanagements, insbesondere des öko-Controllings gesehen werden kann.

Auf der überbetrieblichen Ebene ist der Instrumentenkasten hingegen noch nicht so weit entwickelt. Hier sind zu nennen (6):

- Analysen von Einzelstoffströmen auf nationaler Ebene; Bilanzen über Verbrauch und Verbleib von Blei und Cadmium; Fallbeispiele der EnqueteKommission „Schutz des Menschen und der Umwelt";

nationale Material- und Energieflussrechnungen;

- regionale Stoffstromanalysen, aber auch die Arbeiten auf dem Feld des Industrial Metabolism bzw. der Industrial Ecology;

- bedürfnisfeldbezogene Stoffstromanalysen, z.B. zu den Bedürfnisfeldern Textilien/ Kleidung sowie Bauen und Wohnen und

- Organisationsformen der Kooperation und Kommunikation in Akteursketten des Stoffstrommanagements.

Ökobilanzen stellen ein beide Bereiche überspannendes Instrument dar. In der Frage der Vereinheitlichung ihrer Methode und Durchführung konnten im Zuge der internationalen Normungsarbeit im Rahmen des ISO/TC 207 „Environmental Management" in den letzten Jahren erhebliche Fortschritte gemacht werden.

Das Umweltbundesamt hat eine Methode zur Bewertung in Ökobilanzen entwickelt, die auf drei Kriterien beruht: 
1. potenzielle Gefährdung der ökologischen Schutzgüter in den betreffenden Wirkungskategorien (Ökologische Gefährdung);

2. Abstand des derzeitigen Umweltzustands in den Wirkungskategorien von einem Zustand der ökologischen Nachhaltigkeit oder einem anderen angestrebten Umweltzustand (Distance-toTarget) und

3. Anteil der Umweltbelastungen, die dem Untersuchungsgegenstand zuzuordnen sind, an den jeweiligen Gesamtjahresemissionen in Deutschland (Spezifischer Beitrag).

Die zu diesen drei Aspekten zusammengetragenen Hintergrundinformationen können auch im Stoffstrommanagement zur Operationalisierung der ökologischen Dimension genutzt werden (7).

Insgesamt ist festzustellen, dass die stofflich-naturwissenschaftlichen Analyseinstrumente und -methoden sehr viel weiter fortgeschritten sind als ökonomisch-organisatorische.

\section{Defizite der theoretischen Fundierung}

Der Mangel auf der Methodenebene ist ein unmittelbarer Reflex auf die bestehenden Defizite in der theoretisch-konzeptionellen Fundierung des Stoffstrommanagements. Das Konzept wurde in Deutschland in einem umweltpolitischen Kontext mit natur- und ingenieurwissenschaftlichem Schwerpunkt entwickelt und hat zu einer entsprechenden hohen Ausdifferenzierung in diesen Feldern geführt.

Die konsequente Anwendung von ökonomischen und organisatorischen sowie allgemein systemwissenschaftlichen Erkenntnissen steht dagegen erst am Anfang. Ein Beispiel dafür liegt mit der Arbeit von Spengler zur betriebswirtschaftlichen Planung und Steuerung von Stoff- und Energieströmen im Produktionsunternehmen vor, die eine produktionswirtschaftliche Fundierung bietet (8).

Daneben zeigt der Beitrag von Hummel wichtige Zusammenhänge aus der Perspektive des strategischen Öko-Controlling auf (9). Darin wird ein Controlling-Konzept zur Planung, Steuerung und Kontrolle von Stoffströmen anhand der Dimensionen Ökologie, Kosten und Wettbewerbsdifferenzierung entwickelt, das strategische Richtungssicherheit ermöglicht.

Über diese ersten Ansätze hinaus bestehen interessante Transferpotenziale insbesondere aus folgenden Bereichen:
- Supply-Chain-Management: Das Management von Wertschöpfungsketten beschäftigt sich traditionell mit Materialströmen und bietet damit vielfältige Ansatzpunkte (10). Diese liegen z.B. in der (Kosten- und Mengen-) Optimierung von Materialströmen sowie der Gestaltung der Beziehungen entlang der Wertschöpfungskette zu Kunden und Lieferanten.

- Organisations-/Netzwerktheorie: Beispielhaft sei auf die Arbeiten von Aulinger und Schneidewind zu ökologischen Kooperationen verwiesen, die auf Gestaltungs- und Handlungsmöglichkeiten eingehen (11). Die konzeptionelle Basis bieten verschiedene sozialund wirtschaftswissenschaftliche Theorien, die Hinweise zur erfolgreichen Gestaltung von Akteursnetzwerken geben.

- Ansätze der Systemwissenschaft zur Modellierung komplexer sozio-technischer Systeme: So sind beispielsweise das Wissen der Kybernetik oder des ingenieurwissenschaftlichen Systems Engineering bisher kaum für die Stoffstrommanagementdebatte aufgegriffen worden.

Umweltökonomische Ansätze vermögen zwar einzelne Analyse- und Gestaltungsherausforderungen in Stoffstromketten abdecken, sie geraten jedoch durch die Komplexität der Stoff- und Akteurbeziehungen an Grenzen (12).

Eine weitere Herausforderung besteht darin, Effizienz- und Suffizienzpotenziale zu verbinden. Hierzu muss die Ebene ökologischer Herausforderungen und technischer Lösungsmöglichkeiten mit den aus korrespondierenden Akteursund Interessenkonstellationen resultierenden Möglichkeiten verknüpft werden. In seiner Studie „Nachhaltiges Deutschland“ hat das Umweltbundesamt für wichtige Bedürfnisfelder Szenarien dargestellt (Status-Quo, Effizienz, Struktur- und Bewusstseinswandel), die diese Verbindungen aufzeigen sollen (13).

\section{Das Gesamtverzeichnis des Informationsdienstes "Ökologisches Wirtschaften" finden Sie auf unseren Internetseiten unter:}

\section{http://www.oekom.de}

Wir freuen uns auf Ihren Besuch!
Für den Bereich Bauen und Wohnen ist die Szenariomethode in einem vom Umweltbundesamt finanzierten Forschungsprojekt des Öko-Instituts durch die Entwicklung des EDV-gestïtzten Stoffstrommodells BASiS weiterentwickelt worden (2). Für die Abbildung unterschiedlicher Entwicklungsmöglichkeiten unter Effizienz- und Suffizienzaspekten können sowohl die technischen Alternativen in den Prozessketten, als auch Varianten bei der Nachfrage erfasst werden. Die entsprechenden Stoffströme können den Umweltkategorien Rohstoffbedarf, Emissionen von Treibhausgasen und Säurebildnern, Abfälle/Reststoffe und Flächenverbrauch zugeordnet werden.

\section{- Umsetzung in Praxisprojekten}

Die Gestaltung überbetrieblicher Stoffströme ist heute Thema in einer großen Zahl von Branchen (Textil, Papier, Elektronik, Automobil, z.T. Lebensmittel, Chemie allgemein). Einen guten Überblick gibt der Tagungsband zur Sommerakademie 1998 der Deutschen Bundesstiftung Umwelt zum Thema Stoffstrommanagement (14). Manche Aktivitäten, die ebenfalls durch die beiden zentralen Elemente Stoffstromorientierung über den Produktlebensweg und Akteursorientierung gekennzeichnet sind, haben sich aus anderen Ursprüngen ergeben und werden unter anderen Bezeichnungen durchgeführt. Hierzu gehören Kooperationen, die sich aus Anforderungen des Kreislaufwirtschafts-/ Abfallgesetz oder im Rahmen der integrierten Produktpolitik (15) entwickelt haben.

Es gibt in der Praxis einen bunten, aber immer enger werdenden Flickenteppich an derartigen Projekten. Die Schwierigkeit, diese Projekte dem Stoffstrommanagement zuzuordnen und das Stoffstrommanagement gegenüber anderen Themen wie dem Öko-Controlling oder der integrierten Produktpolitik abzugrenzen, hängt eng mit dem beschriebenen theoretischen und methodischen Defizit einer mangelnden Verknüpfung von strategisch-politischen Zielen und praktischen Handlungsansätzen zusammen.

\section{Die Förderlandschaft}

Abschließend wird kurz skizziert, wer die Motoren für die Förderung von Pilotprojekten sind. Das ursprünglich von der Enquete-Kommission entwickelte Konzept des Stoffstrommanagements ist vom Bundesumweltministerium und dem Umweltbundesamt (UBA) aufgegriffen und in das umweltpolitische Alltagsgeschäft integriert worden. Es wird unter anderem durch Vorhaben 
im Umweltforschungsplan gefördert. Das Fachgebiet Stoffflüsse des UBA ist auf verschiedenen Ebenen an der politisch-strategischen und methodisch-praktischen Weiterentwicklung des Themas beteiligt.

Die Deutsche Bundesstiftung Umwelt hat das Stoffstrommanagement unter anderem als Konzept zur Bündelung von Einzelthemen und zur Verstärkung der überbetrieblichen Perspektive des Umweltmanagements aufgegriffen und beabsichtigt, durch Förderung entsprechender Projekte die Umsetzung bisher noch theoretischstrategischer Ansätze in die Praxis zu fördern.

Das Bundesforschungsministerium hat 1998 mit der Förderinitiative „Modellprojekte für nachhaltiges Wirtschaften“ die bis dahin stark auf Umwelttechnik orientierte Förderpraxis auf stärker systemare Fragestellungen ausgeweitet und explizit einige Projekte zum regionalen oder bedürfnisfeldbezogenen Stoffstrommanagement in die Förderung aufgenommen. Mit dem im November 1999 veröffentlichten Förderschwerpunkt „Integrierter Umweltschutz - Betriebliche Instrumente für nachhaltiges Wirtschaften “ haben diese Anfänge eine konsequente Fortführung gefunden. In den vier Förderfeldern

- informationstechnische Instrumente,

- Controllinginstrumente,

- Planungsinstrumente und

Kommunikationsinstrumente

für nachhaltiges Wirtschaften werden systematisch Aspekte des betrieblichen und betriebsübergreifenden Stoffstrommanagements betont. Einen wertvollen Beitrag zur Förderung des Stoffstrommanagements leisten schließlich Unternehmen wie etwa der Otto-Versand oder der Springer-Verlag, die mit wissenschaftlich begleiteten Pilotprojekten wichtige Impulse für die Gesamtdebatte geben.

\section{Vernetzung der Aktivitäten}

Um die oben skizzierten Herausforderungen zu bewältigen, spielen die Foren, in denen Netzwerkaktivitäten konzeptionell und praktisch vorangetrieben werden, eine wichtige Rolle. $\mathrm{Zu}$ solchen Vernetzungsforen gehören das Umweltbundesamt und die Deutsche Bundesstiftung Umwelt. Im internationalen Kontext sind die bei der SETAC (Society of Environmental Toxicology and Chemistry, die international die Entwicklung der Ökobilanz-Methodik wesentlich vorangetrieben hat) angesiedelten und durch die Ökobilanzvorarbeiten initiierten ChainNet-Aktivitäten zu nennen. Internet-gestützte Communi- ties wie das International Substance Chain Network (,Subchain“) sind gerade im Aufbau (16).

Die Universitäten spielen in dieser Vernetzung bisher eine untergeordnete Rolle, jedoch sind auch hier Entwicklungen im Gange. Hierzu gehören beispielsweise die Schaffung einer eigenständigen Professur „Nachhaltiges Prozessmanagement" an der Universität Bremen, die sich unter anderem der überbetrieblichen Koordination von Stoff- und Materialströmen widmen soll, oder der internationale Studiengang Umweltund Ressourcenmanagement an der TU Cottbus, der sich explizit mit Stoffstrommanagementfragen auseinandersetzt. Daneben steigt die Zahl der Lehrstiihle, die Stoffstrommanagementthemen in ihr Lehr- und Ausbildungsprogramm integrieren.

\section{- Konsequenzen}

Vor dem Hintergrund dieser Analyse sind folgende Prioritäten für die weitere Entwicklung des Themas Stoffstrommanagement zu erkennen:

- Die Kluft zwischen den großen strategischpolitischen Zielen und den vergleichsweise bescheidenen Handlungsmöglichkeiten auf regionaler oder Unternehmensebene muss durch geeignete Modelle und Konzepte auf der Mesoebene verringert werden. Hierzu gehören der Ausbau des Bedürfnisfeldansatzes und die Entwicklung sektoraler Nachhaltigkeitskriterien und -indikatoren mit dem Ziel größerer ökologischer Richtungssicherheit.

Eine weitere methodische Fundierung des Stoffstrommanagements ist wünschenswert. Hochschulen können dabei ein koordinierende Rolle einnehmen und methodische Arbeit leisten.

Insgesamt bedarf es dabei einer noch besseren Vernetzung der Aktivitäten und des Austauschs zwischen den Akteuren.

\section{Anmerkungen}

(1) Enquete-Kommission "Schutz des Menschen und der Umwelt" (Hrsg.) (1994): Die Industriegesellschaft gestalten - Perspektiven für einen nachhaltigen Umgang mit Stoff- und Materialströmen, Bonn 1994.

(2) Vgl. Buchert, M. et al.: Stoffflussbezogene Bausteine für ein nationales Konzept der nachhaltigen Entwicklung in Deutschland, UBA-Texte 47/99, Umweltbundesamt, Berlin 1999.

(3) Vgl. Grießhammer, R.: Am Anfang war das DDT. Vom Schadstoff des Monats zur nachhaltigen Innovation. In: Politische Ökologie, Heft 62, September 1999, S. 24-27. (4) Vgl. Huber, J.: Die Konsistenz-Strategie. Effizienz und Suffizienz alleine können Nachhaltigkeit nicht sichern. In: Politische Ökologie, Sonderheft 11, Januar/Februar 1998, S. 26-29.
(5) Vgl. Hofmeister, S.: Über Effizienz und Suffizienz hinaus. In: Politische Ökologie, Heft 62, September 1999, S. 34-36.

(6) Für eine ausführliche Darstellung und die entsprechenden Literaturverweise vgl. Friege, H. / Engelhardt, C. / Henseling, K. O. (Hg.): Das Management von Stoffströmen. Geteilte Verantwortung - Nutzen für alle. Berlin 1998.

(7) Vgl. Umweltbundesamt (Hrsg.): Handbuch „Bewertung in Ökobilanzen". UBA-Texte 92/99, Berlin 2000.

(8) Vgl. Spengler, T.: Industrielles Stoffstrommanagement Betriebswirtschaftliche Planung und Steuerung von Stoff- und Energieströmen im Produktionsunternehmen, Berlin 1998.

(9) Vgl. Hummel, J.: Strategisches Öko-Controlling - Konzeption und Umsetzung in der textilen Kette, Wiesbaden 1997.

(10) Vgl. Handfield, R. B. / Nichols, E. L.: Introduction to Supply Chain Management, New Jersey 1999.

(11) Vgl. Aulinger, A.: (Ko-)Operation Ökologie - Kooperationen im Rahmen ökologischer Unternehmenspolitik, Marburg 1995, und

Schneidewind, U.: Die Unternehmung als strukturpolitischer Akteur, Marburg 1998.

(12) Für eine ausführliche Auseinandersetzung vgl. z.B. Zundel, S. et al.: Stoffstrommanagement - Zwischenbilanz einer Diskussion. In: Zeitschrift für Umweltpolitik und Umweltrecht 3/98, S. 317-340, insb. S. 333ff.

(13) Umweltbundesamt (Hrsg.): Nachhaltiges Deutschland. Wege zur einer dauerhaft-umweltgerechten Entwicklung. Berlin 1997.

(14) Brickwedde, F. (Hrsg.): Stoffstrommanagement Herausforderung für eine nachhaltige Entwicklung, Tagungsband zur 4. Internationalen Sommerakademie St. Marienthal, Deutsche Bundesstiftung Umwelt, Osnabrück 1999.

(15) Vgl. hierzu Rubik, F./ Simshäuser, U.: Integrierte Produktpolitik: Entwicklung und Perspektiven. In: Ökologisches Wirtschaften 5-6/99, S. 8-9.

(16) Zu "ChainNet" vgl. Wrisberg, N. / de Haes, H. U. / Triebswetter, U. / Eder, P. / Clift, R. (Hrsg.): Analytical tools for environmental design and management in a systems perspective, CHAINNET, Leiden 1999; Zu "Subchain": http://www.uni-oldenburg.de/ produktion/sonstiges/subchain/

\section{Die Autoren}

Dr. Karl-Otto Henseling arbeitet in der Abteilung Stoffflüsse des Umweltbundesamtes (UBA).

Kontakt: UBA, Seecktstr. 6-10, 13581 Berlin. Tel. 030/ 8903-3593, Fax -3232,

E-mail: karl-otto.henseling@uba.de

Dr. Uwe Schneidewind ist Professor für Produktionswirtschaft, Stefan Seuring ist wissenschaftlicher Mitarbeiter an der Carl-von-Ossietzky-Universität oldenburg.

Kontakt: Birkenweg 5, 26111 Oldenburg, Tel. 0441/ 798-8255 (Schneidewind), -8310 (Seuring) Fax -8341, E-mail: Uwe.Schneidewind@uni-oldenburg.de; Stefan.Seuring@uni-oldenburg.de 
(c) 20I0 Authors; licensee IÖW and oekom verlag. This is an article distributed under the terms of the Creative Commons Attribution Non-Commercial No Derivates License (http://creativecommons.org/licenses/by-nc-nd/3.o/), which permits unrestricted use, distribution, and reproduction in any medium, provided the original work is properly cited. 